\title{
An Analytical Model of Web Server Load Distribution by Applying a Minimum Entropy Strategy
}

\author{
Teeranan Nandhakwang, Settapong Malisuwan, Jesada Sivaraks, and Navneet Madan
}

\begin{abstract}
This paper presents an analytical model and the way of simulation for distributing workload on a distributed web server system. The increase in the Internet traffic has also necessitated the conventional Domain Naming Service (DNS) to operate at a much lower efficiency. Among a number of problems associated with the DNS, a key problem has to do with the authoritative DNS not being able to process complete knowledge of the proximity. This makes the authoritative DNS less effective in monitoring server availability and routing incoming requests around failed servers. The workload distribution strategy on the other hand, keeps track of the state and health of the web server. This avoids connection delay due to, for example, a failed server, which can be temporarily by passed by workload distribution. From a modeling standpoint, the conventional DNS assumes equal queue size for each web server in a round-robin setting. Under load balancing, the queue size for each web server differs based on the probability of accessing that server. This probability is based on such factors as the geography, server health, server response, server threshold, session capacities, and the round trip time. In this paper, both conventional and global workload distribution strategies are developed and compared based on a finite set of practical traffic scenarios.
\end{abstract}

Index Terms-Domain name server, entropy, workload distribution strategy, round-robin setting.

\section{INTRODUCTION}

Increasingly companies are turning to World-Wide-Web (WWW) as an option channel for software distribution, online customer service, and business transactions. In the modern day, the role performed of web server is vital to businesses. Successful companies need the capacity to cater to millions of transactions on its server. Initially, several companies reported that they who could not support the colossal volume of transactions specifically on days when stock markets experienced a crash [1].

The phenomenal growth in popularity of the Internet has necessitated internet traffic to be monitored and controlled. This growth has posed the need for research deliberated to decrease the volume of internet traffic originated web users and servers, by using Domain Name Server (DNS). This paper considers a global workload distribution strategy on a distributed web server system [1]-[3].

The most common convention distributed web server

Manuscript received February 4, 2013; revised April 17, 2013.

Teeranan Nandhakwang is with the Security and Military Analyst in Thailand (e-mail: jesada.s@nbtc.go.th).

Settapong Malisuwan is with the Vice Chairman of National Broadcasting and Telecommunications, Bangkok, Thailand.

Jesada Sivaraks and Navneet Madan are with the National Broadcasting and Telecommunications Commission. system is based on round-robin domain name resolution (RR-DNS), which assigns HTML document requests to web server [3]. The round-robin technique is useful specifically in the case that HTTP requests access HTML document of a standard size and the load and service rate of web servers are fairly comparable [4].

Nevertheless, the web servers are heterogeneous systems hence processing nodes are expected to have dissimilar processing speeds. They "can leave and joint the system resource pool at any time" [4]. Therefore dynamic strategies are desirable to balance workload among the web servers [5].

The drawback of the round-robin DNS is that address-caching mechanism permits DNS control on a relatively small portion of the requests. An uneven distribution of clients' requests from diverse number of domains causes imbalance so when many clients from one single domain are assigned to the same single server these results in overload of the web server [3].

\section{System ModeL}

The system is modeled by open queuing network, which is made of numerous interconnected queued servers. An excessive number of requests are entered into the system independently. Therefore this research considers a distributed web server system architecture that uses DNS as a "typical centralized scheduler, applies some scheduling strategy in routing the requests to the most suitable web server" [1]. A well defined and transparent architecture is obtained from a "single virtual interface to the outside world, at the least at URL level" [4], as shown in Fig. 1.

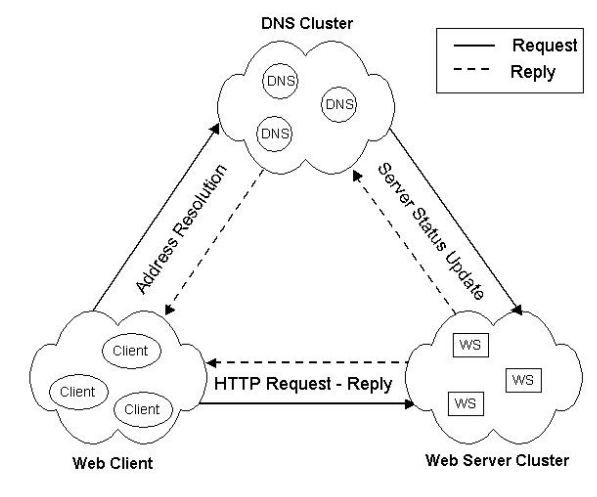

Fig. 1. System model and network flow.

Network is fault free and strongly connected. However, the traffic of the network is various. It depends on the request from web client. Also, web servers can leave and join the system resource pool at any time [8]. They are no communication between web servers. 
This research denotes the average page response time $E[R]$ by. This may write $E[R]$ as the sum of the average total waiting time, the average communication delay, and the average DNS synchronization; that is,

$$
E[R]=E[W]+E[D]+E[S]
$$

where $E[W]$ denotes the average waiting time, $E[D]$ denotes the average communication delay, and $E[S]$ denotes the average DNS synchronization.

To understand workload behavior on the distributed web server system (physical or logical connection), the research adds two fictitious stations to the network: request and receive. The request station generates all external arrival and the receive station receives all customers leaving the web server cluster. This is shown in Fig. 2.

This paper uses $q_{e i}\left(\sum_{i=1}^{M} q_{e i}=1\right)$ [5], [6], and [7] to denote the probability that an external arrival is directed to web server $i$. Similarly, this paper uses $q_{i o}$ to denote the probability of the task that finishes it services at web server $i$ and leaves the web cluster. From [5], [8], [9], [10], [11], and [12], the traffic equation for the network is given by: $\lambda_{i}=\gamma_{i}+\sum_{j=1}^{M} \lambda_{i} q_{j i}$. Where $\lambda_{i}$ denotes the average arrival rate at web server $i, \gamma_{i}$ denotes the external arrival into web server $i$ from a Poisson stream $\left(\gamma_{i}=\gamma q_{e i}\right)$. Then $\gamma=\sum_{i=1}^{M} \gamma_{i}$.

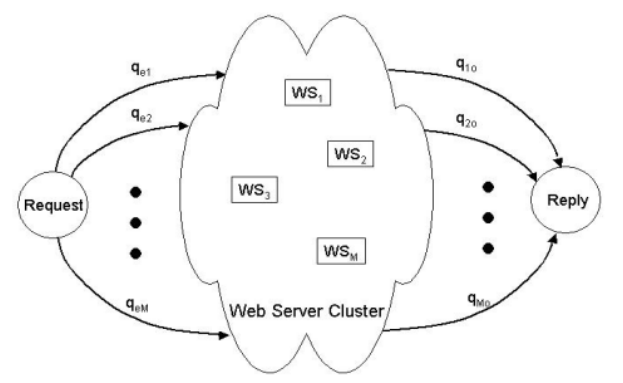

Fig. 2. Represent of an open web server queuing network.

This paper uses $q_{i j}$ to denote the routing probability that on leaving web server ${ }_{i}$ to web server $j$. However, this model, the web servers do not communicate to each other. Therefore, $q_{i j}=0$ and $\lambda_{i}=\gamma_{i}$. It follows [7], [10], and [14] that the average waiting time in the network is given by:

$$
W_{i}=\frac{1}{\mu_{i}\left(1-\rho_{i}\right)}
$$

where $\mu_{i}$ is the service rate, and $\rho_{i}$ is the traffic intensity. The utilization of web server $i$ is given by $U_{i}=\rho_{i}$. In this model, the service rates of the web server are load-dependent [11] and [15]. Then $\mu_{i}=\mu_{i}^{\circ} C_{i}(n)$, where $\mu_{i}^{\circ}$ is called the basic rate service rate. This research uses $C_{i}(n)$ to denote the capacity function where $n$ denotes population vector, and by definition $C_{i}(1)=1$. Using (2) therefore:

$$
E[W]=\sum_{i=1}^{M} \frac{\lambda_{i}}{\gamma}\left[\frac{1}{\mu_{i}\left(1-\rho_{i}\right)}\right]
$$

The average communication delay and the average DNS synchronization time are the average time that packet spent waiting for using channel. They are given by [8] and [11]:

$$
E[T]=\sum_{i=1}^{M} \frac{\lambda_{i}}{\gamma}\left[\frac{1}{x K_{i}\left(1-\lambda_{i}\right)}\right]
$$

where $E[T]$ is the average message delay, $1 / x$ is the average length of a data packet, and $K_{i}$ is the capacity channel at channel $i$. Using (4), then

$$
E[D]=\sum_{i=1}^{M} \frac{\lambda_{i}}{\gamma}\left[\frac{1}{x_{D} K_{D i}\left(1-\lambda_{i}\right)}\right]
$$

and

$$
E[S]=\sum_{i=1}^{M+N} \frac{\lambda_{i}}{\gamma}\left[\frac{1}{x_{S} K_{S i}\left(1-\lambda_{i}\right)}\right]
$$

where $1 / x_{D}$ denotes the average length of a data packet that send and receive between web server and web client, $K_{D i}$ denotes the capacity channel at channel $i$ between web server and web client, $1 / x_{S}$ denotes the average length of a data packet that send and receive between DNS and web server, $K_{S i}$ denotes the capacity channel at channel $i$ between DNS and web server, and $N$ denotes number of DNS in DNS cluster. This research assumes the capacity channel between DNS to web server and web client to web server are equal, also the average length of a data packet from web server to web client and web server to DNS are equal. Using (1), (3), (5), and (6), therefore:

$$
E[R]=\sum_{i=1}^{M} \frac{\lambda_{i}}{\gamma_{D}}\left[\frac{x_{D} K_{D i}\left(1-\lambda_{i}\right)+2 \mu_{i}\left(1-\rho_{i}\right)}{\mu_{i} K_{D i}\left(1-\lambda_{i}\right)\left(1-\rho_{i}\right)}\right]+\sum_{j=1}^{N} \frac{\lambda_{j}}{\gamma}\left[\frac{1}{x_{S} K_{S j}\left(1-\lambda_{j}\right)}\right]
$$

Therefore (7) is "The average page response time corresponds to interval elapsed between the submission of the web client request for given page and the arrival at the client of all objects corresponding to the page request. It includes Transmission Control Protocol (TCP) establish connection time, delays at web server, network transmission time" [5].

\section{A MINIMUM ENTROPY STRATEGY FOR WEb SERVER LOAD DISTRIBUTION}

The uncertainty equation is derived for this group of objects which is an equation from Shannon. The information theoretic heuristic is analyzed at each decision level thus, decreasing the lower bound for the sub problem. In 1948, a measure of uncertainty of a discrete stochastic system known as entropy was introduced [8], [9], and [14]. For a probability distribution $p$ define on a finite set $[n]=\{1, \ldots, n\}$ the Shannon entropy of $\mathrm{p}$ is defined by with the convention that $0 \log 0=0$.

$$
H_{s}(p)=-\sum_{k=1}^{m} P(i) \log P(i)
$$

Entropy is now an essential concept in decision making. 
Additionally, entropy is a constructive information theory which is generally adopted in the information theory is used as a measure for the uncertainty of a discrete probability density function. Since this definition is similar to the one used in statistical mechanics, this measure of uncertainty is labeled entropy. When all probabilities are equal, the entropy reaches its maximum or minimum.

Entropy is adopted in the case that the decision matrix for a set of options contains a certain amount of information. Therefore, entropy is adopted as a tool in certainty level evaluation. Entropy is most practical in investigate the differences among data sets. Although entropy has increasingly adopted in these past years for a variety of cases in information theory, it has great potential as a decision-making tool until now this has not been fully exploited. This study underlines the functional capability of entropy which includes the derivation of probability distributions.

This research proposes a strategy that aims at improving the performance of distributed load to web servers that is likely to perform poorly scheduling algorithm RR-DNS. The proposed scheduling strategy uses information from each web server to make the decision in the mapping of URL names to IP-address of web servers, as illustrated in Fig. 3. This decision problem is solved with a greedy technique, which is used the entropy function as weighted linear function to decide the best web server. The entropy function is an uncertainty measure at the web server for the decision in each request.

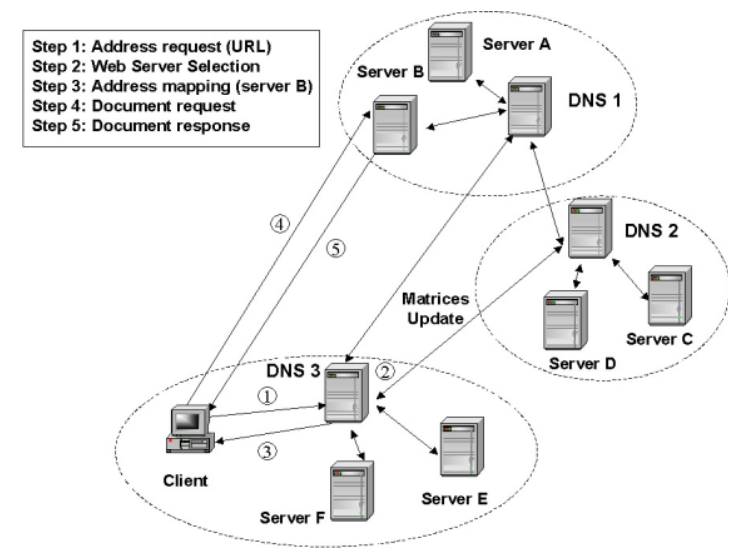

Fig. 3. DNS-Based web server load balancing with minimum entropy policy.

Let $H_{i j}$ be entropy of a web server $i$ with server matrices $j$, and let $x_{i j k}$ be a set of mutually exclusive $m$ events of web server $i$ with server matrices $j$. The entropy function of web server $i$ with server matrices $j, H_{i j}\left(X_{k}\right)$, is defined as

$$
H_{i j}\left(X_{k}\right)=-\sum_{k=1}^{m} P_{i j k}\left(x_{i j k}\right) \log P\left(x_{i j k}\right)
$$

In this paper, the server matrices (e.g., TTL, health condition, session capacity threshold, round-trip time, and geographical region) are mutually exclusive events, then [8] and [14]

$$
H_{i j}\left(X_{1}, \ldots, X_{k}\right)=H_{i j}\left(X_{1}\right)+H_{i j}\left(X_{2}\right)+\ldots+H_{i j}\left(X_{k}\right)
$$

$$
H_{i j}\left(X_{1}, \ldots, X_{k}\right)=-\sum_{j=1}^{n} \sum_{k=1}^{m} P\left(x_{i j k}\right) \log P\left(x_{i j k}\right)
$$

Therefore

$$
\operatorname{OPT}\left(H_{i j}\right)=\min _{i}\left(\sum_{j=1}^{n} H_{i j}\left(X_{1}, \ldots, X_{k}\right)\right)
$$

where $O P T\left(H_{i j}\right)$ is the web server that has minimum entropy. It follows [16], the entropy function is minimum, in fact equal to zero, when its values is certain, an observation can yield a good condition on the web server. Similarly, the entropy function maximum when the random variable is uniformly distributed; the outcome of the web server has maximum uncertainty. This research measures entropy level by server matrices from each web server to calculate the desired relative workload distribution workload between the distributed web servers. Fig. 4 shows an example of certainty level in each web server. The workload will be assigned to the web servers are depended on the entropy. The web server with smaller entropy will be assigned workload more than the web server with higher entropy.

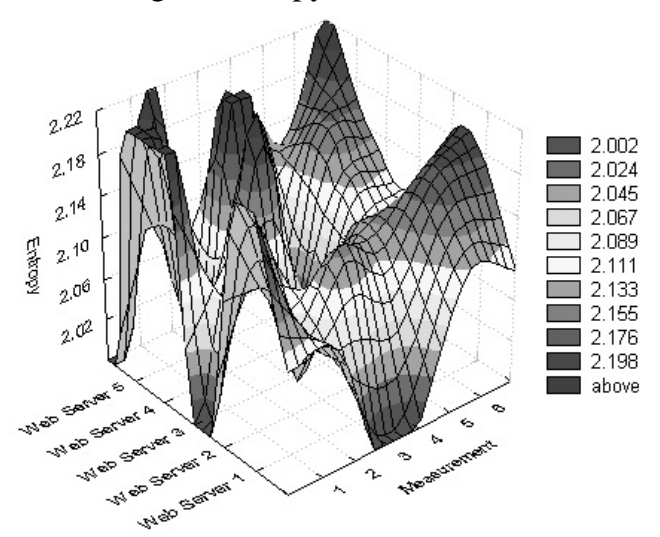

Fig. 4. Web server entropy measurement.

\section{PARAMETER AND EXPERIMENTS DESIGN}

This section presents the experiment with their base value and distributions that characterize the entire system and the DNS with minimum entropy policy. Table I summarizes the system and workload parameters that compare the performance of the minimum entropy load balancing strategy with the traditional RR-DNS, the RR- DNS [3] and [10]. HTML document response time is a major index to measure the performance of the load balancing strategy. In particular, looking at the minimum average page response time, this can deduce whether the load balance or not. Hence, the performance of several scheduling policies is evaluated by focusing on the minimum page response time observed during the simulation runs.

This experiment divides global DNS cluster into small regions. When a client requests the HTML document from web server, it sends the query to intermediate name server. Both Intermediate name server and client is the same region, and assume that the number of hop to reach web server from DNS and client are similar. Entire system operates with no cache. Each DNS, the minimum entropy load balancing policy, sends the queries to each web server and other DNS for updating their information. Server metrics on each DNS 
contains with web server round trip time, TTL to reach the web server, and response time.

TABLE I: PARAMETERS OF THE SYSTEM

\begin{tabular}{|l|l|c|}
\hline Category & Parameter & Value(default) \\
\hline DNS & Number of DNS & 3 \\
\hline Web Server & $\begin{array}{l}\text { Number of Web } \\
\text { Server }\end{array}$ & 5 \\
\hline Workload & Basic Service Rate & $\begin{array}{c}400-450 \\
\text { packet/second }\end{array}$ \\
\hline & $\begin{array}{l}\text { Number of HTTP } \\
\text { Requests }\end{array}$ & 1000 \\
\hline Server Matrices & Documents Size & $10-100$ packets \\
\hline & Round Trip Time & $32-500 \mathrm{~ms}$. \\
\hline & Response Time & $1-10 \mathrm{~ms}$. \\
\hline & TTL & $64-254$ \\
\hline
\end{tabular}

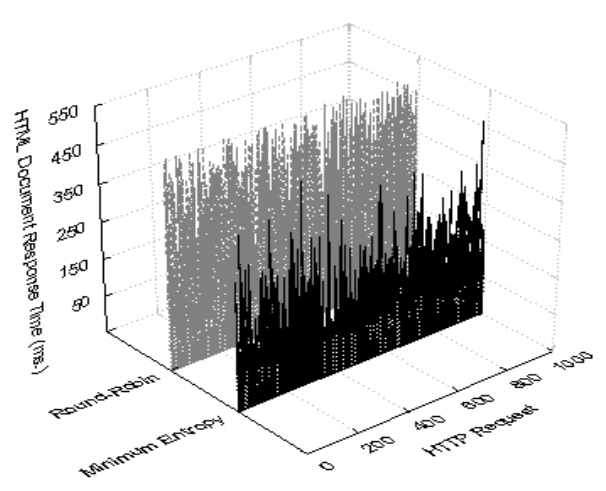

Fig. 5. 1000 HTML documents average response time.

This research uses load distribution and cumulative distribution of the HTML document response time as the performance criterion, because in a highly variable system it is more significant than average values [4] and [15]. For the performance evaluation of proposed policy, this research carried out a large number of experiments. The gold is to measure how effectively the minimum entropy load balancing that controls only a very small percentage of address resolution requests can minimize the workload of the distributed web server. Fig. 5 shows the HTML document average response time of each strategy in 1000 HTTP document requests. The average of the HTML document response time of the minimum entropy strategy is less than the RR-DNS.

TABLE II: THE EXPERIMENT STATISTICAL VALUES

\begin{tabular}{|c|l|r|r|l|l|}
\hline Policy & Average & Minimum & Maximum & Variance & Std.Dev \\
\hline $\begin{array}{c}\text { Minimum } \\
\text { Entropy }\end{array}$ & 112.694 & 33.002 & 487.002 & 5997.33 & 77.4424 \\
\hline $\begin{array}{c}\text { Round-Ro } \\
\text { bin }\end{array}$ & 262.674 & 38.002 & 510.002 & 18758.15 & 136.9604 \\
\hline
\end{tabular}

Fig. 6 shows load distribution of 1000 HTML document request on minimum entropy and round-robin strategy. In the minimum entropy policy, 40-percent of the requests are between $100-150 \mathrm{~ms} ., 14$-percent of the request are between $50-100 \mathrm{~ms}$., and 46-percent are greater than $150 \mathrm{~ms}$. In the other hand, round-robin policy, 12-percent are between $100-$ $150 \mathrm{~ms}$. and $450-500 \mathrm{~ms}$., 4-percent of the requests are between 50 - $100 \mathrm{~ms}$., 11-percent are greater than $500 \mathrm{~ms}$. and the rest are spread from $150 \mathrm{~ms}$. to $450 \mathrm{~ms}$.

Fig. 7 compares the cumulative distribution for the DNS proximity using minimum entropy strategy and traditional
DNS with round-robin strategy. This figure shows that both policies guarantee that the maximum HTML document response time is below $550 \mathrm{~ms}$. The 90-percentile of the minimum entropy policy is less than $225 \mathrm{~ms}$ and the 50-percentile is below $125 \mathrm{~ms}$. The RR policy has slightly worse performance with the 90- percentile is less than 450 ms., and the 50-percentile is less than $275 \mathrm{~ms}$. The propose method can improve the average response time much further by tuning the web server matrices to the workload level. To archive this goal, the experiment needs to monitor the server matrices, choosing minimum entropy web server to assign the heavily load and choosing maximum entropy web server to assign light load.

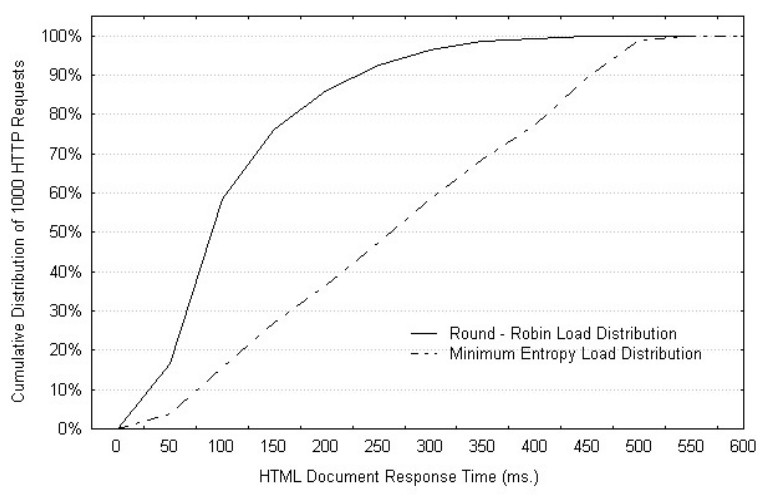

Fig. 6. A minimum entropy load distribution strategy and Round-Robin strategy.
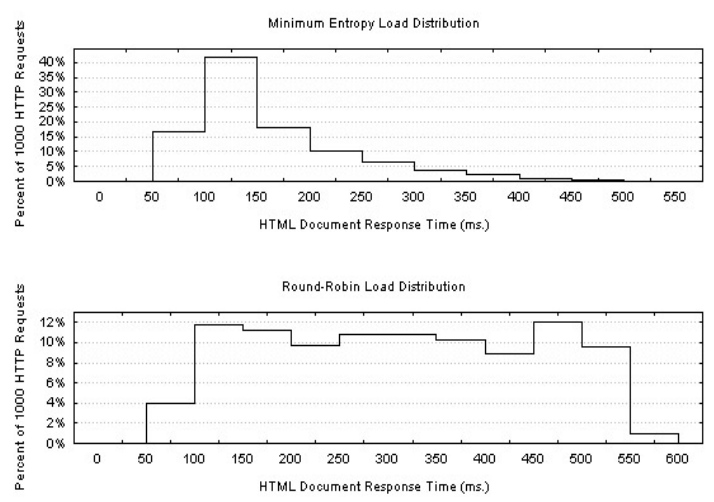

Fig. 7. Cumulative distribution of HTML document average response time.

\section{CONCLUSION}

In this paper, the experiment modified the traditional scheduling strategy to the Domain Name Server (DNS), proposed new policy, and studied the impact for a diverse range of scenarios. The new approach proposed is based on the entropy function of the web server matrices (e.g., health condition, session capacity threshold, round-trip time, and geographical region). The tradition round-robin DNS (RR-DNS) policy is not applicable to network with dynamic traffic scenarios. The experiments of new policy showed that they are able to providing suitable web server in each request to the web client. The entropy strategy can be useful for analyzing and modifying an existing system, and assessing other newly proposed strategies. In the near future, the experiment will improved the technique to distributing workload on the distributed web server system whether local 
or global connection. The research is also going on building a simulation environment to have more validation for the obtained results.

\section{REFERENCES}

[1] M. Colajanni, P. S. Yu, and D. M. Dias, "Analysis of task assignment policies in scalable distributed web-server systems," in Proc. IEEE Transactions on Parallel and Distributed Systems, vol. 9, no. 6, pp. 585-600, 1998.

[2] V. Cardellini, M. Colajanni, and P. S. Yu, "Geographic load balancing for scalable distributed web systems," in Proc. The 8th International Symposium on Modeling, Analysis and Simulation of Computer and Telecommunication Systems, 2000.

[3] V. Cardellini, "Dynamic load balancing on web-server systems," in Proc. IEEE Internet Computing, vol. 3, no. 3, pp. 28-39, 1999.

[4] D. Andresen, T. Yang and O. Ibarra, "Toward a scalable distributed WWW server on workstation clusters," Journal of Parallel and Distributed Computing, 1997.

[5] P. S. Yu, "Geographic load balancing for scalable distributed web systems," in Proc. 8th International Symposium on Modeling Analysis and Simulation of Computer and Telecommunication Systems (Cat No PR00728) MASCOT-00, 2000.

[6] C. Lu and S. Lau, "An adaptive load balancing algorithm for heterogeneous distributed systems with multiple task classes," in Proc. The 16th International Conference on Distributed Computing Systems, pp. 629-636, 1996.

[7] R. Nelson, "Probability, stochastic processes, and queueing theory: The mathematics of computer performance modeling," Springer-Verlag New York, Incorporated, 1995.

[8] A. A. A. Sveshnikov and R. A. Silverman, "Problems in probability theory, mathematical statistics and theory of random functions," Dover Publications, Incorporated, 1987.

[9] J. Watts and S. Taylor, "A practical approach to dynamic load balancing," in Proc. IEEE Transactions on Parallel and Distributed Systems, vol. 9, no. 3, pp. 235-248, 1998.

[10] P. G. Harrison and N. M. Patel, "Performance modeling of communication networks and computer architectures," International Computer Series, Addison Wesley Longman, Inc., 1992.

[11] K. Kant and M. M. Srinivasan, Introduction to Computer System Performance Evaluation, McGraw-Hill Higher Education, 1992.

[12] L. Kleinrock, Queuing systems: Theory, vol. I, Wiley, John and Sons, Incorporated, 1975.

[13] L.Kleinrock, Queuing systems: Theory, vol. II, Wiley, John and Sons, Incorporated, 1976.

[14] T. G. Robertazzi, Computer Networks and Systems: Queuing Theory and Performance Evaluation, Springer-Verlag New York, Incorporated, 2000.

[15] R. Jain, The art of computer systems performance analysis: Techniques for experimental design, measurement, simulation, and modeling, Wiley, John and Sons, Incorporated, 1990.

[16] M. Li and P. Vitanyi, An Introduction to Kolmogorov Complexity and Its Applications, Springer-Verlag New York, Incorporated, 1997.

[17] H. Kameda, J. Li, C. Kim, and Y. Zhang, Optimal Load Balancing in Distributed Computer Systems, Springer-Verlag New York, Incorporated, 1997.
Teeranan Nandhakwang was born in Thailand. He received his $\mathrm{PhD}$ in Optimizing Web Server Load Distribution with Evolutionary Computation from Florida Institute of Technology, USA in 2003. He received his MS. In Engineering Management from Florida Institute of Technology, USA and completed his BSc from King Mongkut's Institute of Technology Ladkrabang, Thailand in 1998. He currently works as a Security and Military Analyst in Thailand. His previous appointments were as a counter-terrorist assault team leader, System Analyst Officer, Command and Control System's Network Engineer, Instructor at Peace Operations Center, and Research Fellow at Strategic Studies Center. He joined the National Defense College, Royal Thai Armed Forces Head Quarters in 2010, and is the Deputy Director of Political and Military Affairs Division.

Settapong Malisuwan was born on $24^{\text {th }}$ March 1966 in Bangkok, Thailand $\mathrm{He}$ received his $\mathrm{PhD}$ in electrical engineering (telecommunications), specializing in mobile communication systems from Florida Atlantic University (State University System of Florida), Boca Raton in 2000. He received an MSc in electrical engineering in mobile communications system, from George Washington University in 1996, an MSc in electrical engineering in telecommunication engineering from Georgia Institute of Technology in 1992 and a BSc in electrical engineering from the Chulachomklao Royal Military Academy, Nakhon-Nayok, Thailand in 1990. He served in the Royal Thai Armed Forces for more than 25 years and is currently the Vice Chairman of National Broadcasting and Telecommunications, Bangkok, Thailand. His research interests are in efficient spectrum management and Telecommunications policy and management in Thailand. Col. Dr. Settapong Malisuwan Settapong Malisuwan is currently the Elected Vice Chairman and Board Member in the National Broadcasting and Telecommunications Commission, Thailand

Jesada Sivaraks was born on $12^{\text {th }}$ May 1970 in Bangkok, Thailand. He received his MSEE degree from Oklahoma State University in 1996 and BEng from King Mongkut"s Institute of Technology, Thailand. He completed his $\mathrm{PhD}$ in electrical engineering at Florida Atlantic University, Boca Raton, FL in 2001. Since 2011, he has been working in National Broadcasting and Telecommunications Commission as the Secretary to the Vice Chairman. His PhD work is on the system aspects of Bluetooth, WLAN and Mobile IP/CDPD. His current research interests are in telecommunication planning and related system analysis and efficient spectrum management. He is a member of Tau Beta Pi, Florida Epsilon and was an Honorary Advisory's Chairman of Science \& Technology committee of Parliament in 2009

Navneet K. Madan was born in Bangkok, Thailand on $22^{\text {nd }}$ April, 1987. She received her Bachelor of Business Administration in international business management from Mahidol University in 2008, and received Master of Science degree in strategic management and marketing, Middlesex university, London, United Kingdom. She has been working as an Assistant to Vice Chairman in National Broadcasting and Telecommunications, Bangkok, Thailand since January 2012. Her research interests are in Spectrum Management Strategic Flexibility, Market Orientation and Environmental Uncertainty in Fast Clockspeed Industries 